\title{
GLYCOGEN STORAGE DISEASE IN A MEXICAN BABY GIRL. ULTRAESTRUCTURAL AND LIGHT MICROSCOPY FINDINGS.
}

\author{
*Dr. Marco A. Ponce-Camacho, ** Dr. Juan Pablo Flores-Gutierrez **MC Enrique \\ Ramírez-Bon, ** Dr. Jesús Ancer Rodríguez.
}

*Departamento de Anatomía Patologica y Citoptologia Hospital y Clinica OCA S.A. de C.V. Pino Suarez 645 Nte. Col. Centro Monterrey N. L., México.

**Departamento de Anatomia Patológica Hospital Universitario Dr. Jose Eleuterio Gonzalez, Av. Madero y Gonzalitos Col. Mitras Centro Monterrey N.L., México.

Congenital inborn error of the metabolism are a wide group of diseases that fall in several categories from a surgical pathology point of view. The diagnosis of these diseases requires tissue for biochemical and histology examination. Although some of these diseases are diagnosable by light microscopy, others need special stains and electron microscopy.

We describe a case of Glycogen Storage Disese (GSD) of a six month old baby girl admitted to our Hospital because growth retardation, hypoglycemia and hepatomegaly. She underwent a liver biopsy for light microscopy, ultraestructure and potential enzymatic assay. Hematoxylin and Eosin stained sections showed hepatocytes with a vegetable cell appereance and cytoplasmic granularity. Special stains proved the intracytoplasmic material to be PAS positive diastase sensitive. Ultrastructural findings revealed intracytoplasmic lipid vacuoles and a large amount of glycogen. Light and electron microscopy findings were consistant with GSD. After confirmation of GSD, frozen liver sample was sent to Duke University Biomedical Mass Spectrometry Laboratory for GSD enzymatic assay. The final diagnosis was GSD type III.

GSD includes at least 10 distinct disorders, each of which has a specific enzymatic defect in glycogen metabolism thus leading to an excessive and abnormal glycogen accumulation in different organs such as the liver, heart and skeletal muscle. Diagnosis of these entities is based upon the clinical picture, lab test, light and electron microscopy. Although biochemical frozen tissue examination is needed for confirmation, we believe that electron microscopy is a powerful tool when dealing with a biopsy under suspected metabolic disease because it helps in decide the biochemical assay to perform.

\section{References.}

1.- Fernandes, J. The history of the glycogen storage disease. (Letter) Europ. J. Periapt1995, 154: 423-424.

2.-Mc Adams Aj, Hug G, Bove KE. Glycogen storage disease type I-X, Hum Pathol 1974;5:463

3.- Inborn errors of the metabolism in Stocker \& Denher Pediatric Pathology volume one Second Edition, pages 159-193.

4.- Siciliano M, De Candia E, Ballarin S, Vecchio FM, Servidei S, Annese R, Landolfi R, Rossi L. Hepatocellular carcinoma complicating liver cirrhosis in type IIIa glycogen storage disease. J Clin Gastroenterol $2000 ; 31(1): 80-2$ 

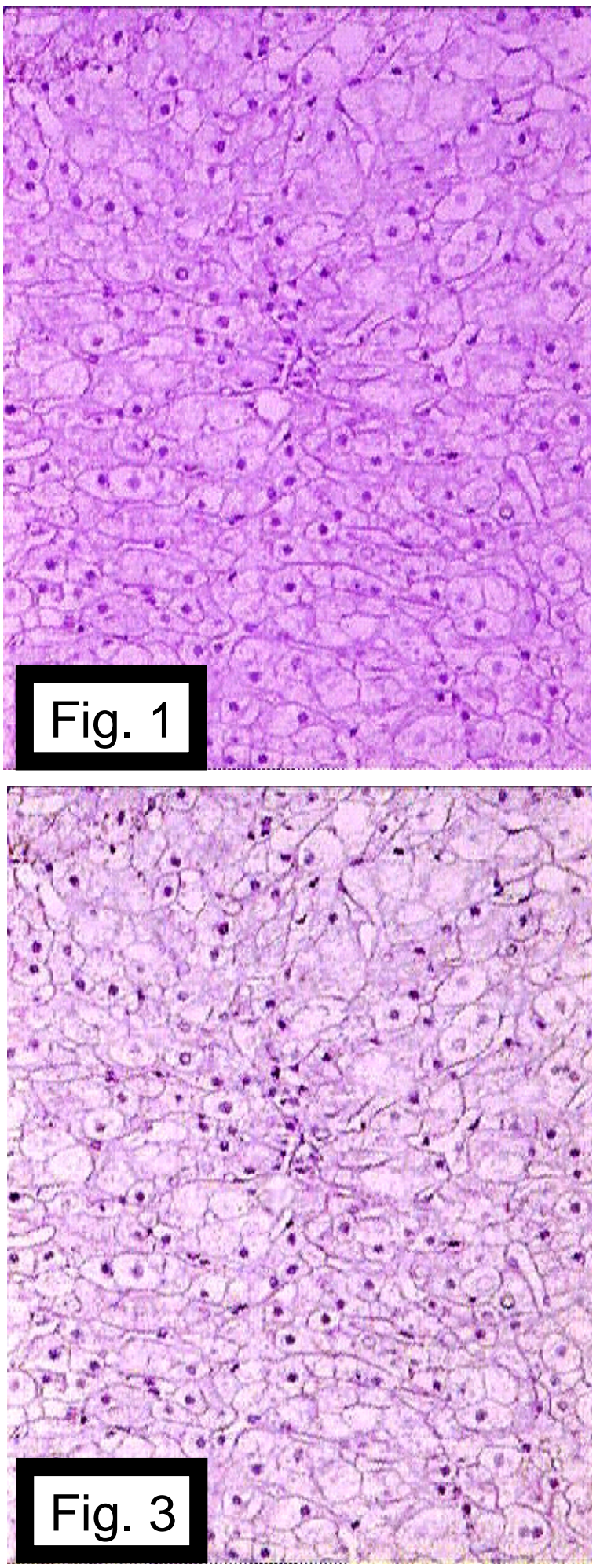
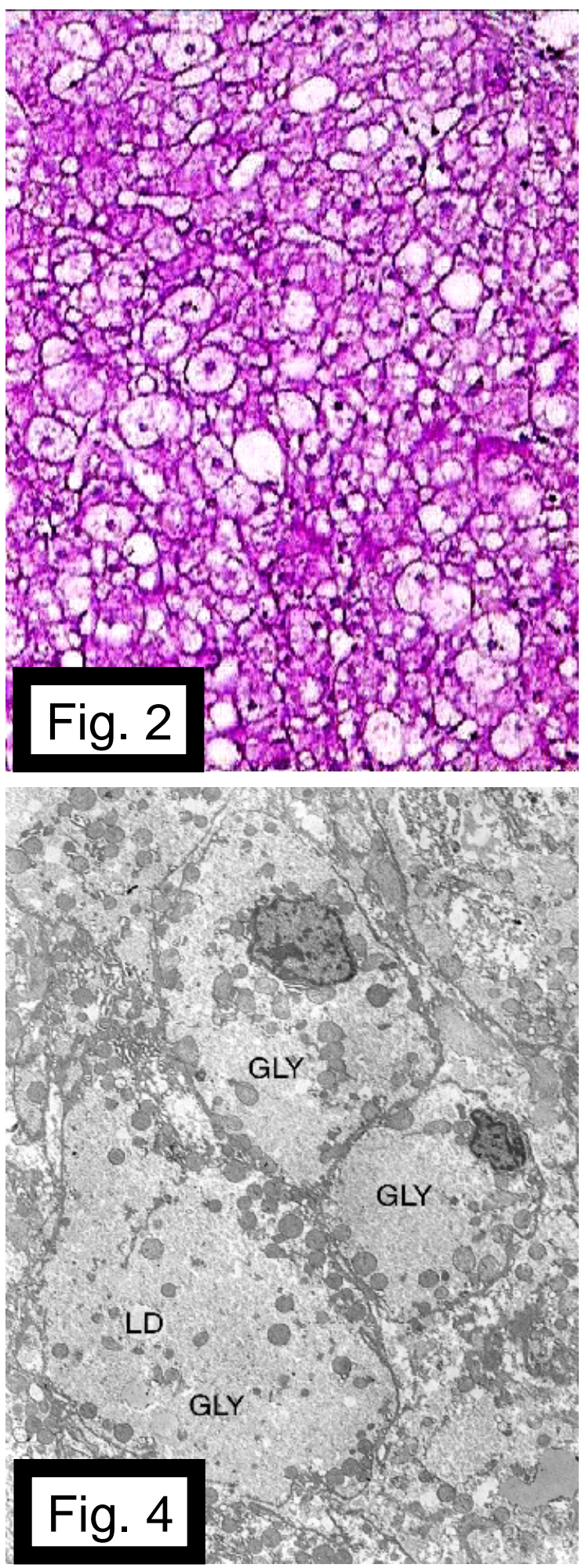

Figure 1: Liver H \& E 10x shows swollen hepatocytes with cytoplasmic granularity.

Figure 2: PAS stain: granular intracytoplasmic material PAS positive.

Figure 3: PAS diastase stain. Intracytoplasmic material is PAS diastase sensitive.

Figure 4: Electron microscopy showing swollen hepatocytes, intracytoplasmic lipid vacuoles (LD) and abundant glycogen granules (GLY). 